\title{
Fibre Architecture of the Deltoid Muscles in the Japanese Macaque, with Special Reference to the Relationship with That of the Pectoral Muscles
}

\author{
KEN FUJINO \\ Department of Laboratory Animal Science, \\ Tokyo Metropolitan Institute of Gerontology, \\ Sakaecho, Itabashi, Tokyo 173, Japan
}

Received May 6, 1993

\begin{abstract}
Spatial fibre architecture of the deltoid muscles in five Japanese macaques was observed and functionally analysed to compare it with that of the adjoining pectoral muscles. The fibre bundles of the deltoids possess a twisted and radiating architecture, as do those in the pectorals, and are basically arranged symmetrically about the central fibre of the acromiodeltoid. Unlike those of the pectorals, the bundles of deltoids do not exhibit any crossing structure. These structural features of the deltoids are thought to have been formed through evolutionary modifications in the mammal-like reptiles and primitive mammals, which have disposed the humerus more perpendicularly while simultaneously preserving the deltoids as a single-joint muscle. The lateral shift in the cleidodeltoid and the accompanying clavicular origin of the pectoralis in brachiators and human are suggested to have been formed concomitantly with the flattening of the thorax as one of the evolutionary traits in primates.
\end{abstract}

Key Words: macaque, deltoid muscle, fibre architecture, functional anatomy, evolution

\section{INTRODUCTION}

Both the $\mathrm{Mm}$. deltoidei and $\mathrm{Mm}$. pectorales are innervated by branches from the brachial plexus and thus are classified into the pectoral limb muscle group. Within the group, however, the two are phylogenetically distant from each other: the former belongs to the dorsal division, and the latter to the ventral (Howell, 1936).

Despite their comparatively distant relationships in phylogeny, the two appear to have retained close relations both in topography and in morphology through evolutionary stages. A survey of the comparative anatomical literature dealing with these muscles in some primitive animals (Sphenodon, Fürbringer, 1900; Echidna, Westling, 1889; Ornithorhynchus, Coues, 1871; Echidna and Ornithorhynchus, McKay, 1895; Didelphys, Coues, 1872) shows that although the pectoral girdle has undergone marked evolutionary modifications (for details, see DISCUSSION), these two muscles have maintained the following relationships: The two are each arranged in a radiating manner, as the most superficial layer over the lateroventral aspect of the 
thorax, and adjoin each other both at the origin and the humeral insertion, the former generally arising from the shoulder girdle cranially whereas the latter arises from the sternal bones caudally.

As part of the evolutionary morphology of the muscles, it would be of great value to elucidate how and why the topographical and the morphological relations between these two muscles have developed and been preserved.

Existing information regarding the morphological and functional relations between the two muscles is limited and fragmentary at best. Saar (1903) attempted to elucidate their topographical and phylogenetical relations in several mammals. Parsons (1898) and Horejsi (1967) have independently suggested the presence of a mutually analogous fibre arrangement (i.e., the presence of fibre twisting) in the two muscles. Inman et al. (1944) have referred to their synergistic electromyographic action in humans.

The purpose of the present study is, therefore,

1. to describe precisely the spatial fibre architecture of the deltoid muscles,

2. to elucidate the morphological and functional implications of this architecture, and

3. to consider the morphological evolution of the deltoid muscles, especially in comparison with that of the pectoral muscles (cf. Fujino et al., 1990).

\section{MATERIALS AND METHODS}

Five male adult Japanese macaques (Macaca fuscata) were examined. Subjects were sacrificed using an overdose of sodium pentobarbital. After the removal of the inner organs, cadavers were immersed in $10 \%$ formalin for fixation with the forelimbs placed at their resting position, i.e., along the lateral side of the thorax. General anatomical observations were then made on the right-side deltoid muscles, namely, Mm. spinodeltoideus, acromiodeltoideus and cleidodeltoideus. The nomenclature of Howell and Straus (1961) was adopted in the present study.

Next, each muscle was excised at both attachments, and immersed in a 15-20\% sulfuric acid solution to soften the connective tissue after the technique of Sacks and Roy (1982). After rinsing the muscle in water and carefully removing blood vessels and excess connective tissue, the author observed the spatial fibre arrangement, paying particular attention to the relation between muscle fibres and tendon.

\section{OBSERVATIONS}

\subsection{General description of the three deltoid muscles}

\subsubsection{M. spinodeltoideus (Figs. 1b, c, d, 2a)}

This muscle arises, mostly by tough aponeurosis but partly (at its ventral end) by fleshy fibres, from the entire caudal edge of the crest of the scapular spine except 
the narrow region adjoining the acromion process. This aponeurosis keeps strongly adhering to the fascia of the infraspinatus muscle lying deep, just before the aponeurosis begins to originate muscle fibres along an arched line. Thus, this arched line on the fascia can actually be regarded as the functionally significant origin (fso, Figs. 1b, d), because the tension exerted by the fibres acts along this line rather than the scapular spine. Toward the humerus, the fibre fasciculi of the spinodeltoid, most of which arise semi-circularly from the deep surface of the aponeurosis, converge and rotate. Near insertion, they become covered by the dorsal fibres of the acromiodeltoid and simultaneously sandwiched by two tendon plates (a deeper, short plate, and a superficial, broad and stout plate). The dorsal fibres of the spinodeltoid attach to the superficial face of the deep plate, while the ventral fibres attach to the proximal half of the deep face of the latter (the dorsal fibres of the acromiodeltoid attach across the entire superficial face of the latter; thus, this tendon plate is common to the two muscles; see below). About $1 \mathrm{~cm}$ before the insertion this common plate is joined by the deep plate and inserts strongly upon the lateral edge of the distal half of the deltoid crest of the humerus. The precise insertion covers the region between 22 to $38 \%$ of the humerus on average (where the proximal end of the humerus is set at $0 \%$ and the distal end at $100 \%)$.

\subsubsection{M. cleidodeltoideus (Figs. la, c, d, 2a)}

This muscle originates from the middle half to three-fifths of the caudoventral aspect of clavicle bone, and is adjoined caudally by the entire insertion of $\mathrm{M}$. subclavius and laterally by the insertion of M. trapezius. Superficial fibres arise from a semicircular tendinous sheet covering this muscle at the origin. Fibre fasciculi appear to run parallel to one another, but in fact they rotate slightly in the opposite direction from those of the spinodeltoid muscle. Insertion is mostly by fleshy fibres and only slightly by thin tendon plate (for its most lateral fibres) upon the medial edge of the distal half of the deltoid crest between 18 to $45 \%$, covering nearly the entire insertion of the sternocapsular portion of the pectoralis major (cf. Fujino et al., 1990).

\subsubsection{M. acromiodeltoideus (Figs. la, b, c, 2a)}

The deeper portion of this muscle arises from the caudoventral aspect of the acromion process by fleshy fibres, while the superficial portion arises from a tendinous sheet widely covering the muscle. The origin occasionally extends onto the lateral tip of the clavicle just below the insertion of $\mathrm{M}$. trapezius. In contrast to the other two deltoid muscles, the fibres in this muscle run nearly parallel with one another, although fibres from the dorsal and the ventral sides (especially the former) tend to converge and go beneath the main portion of the muscle belly. Most of 
a

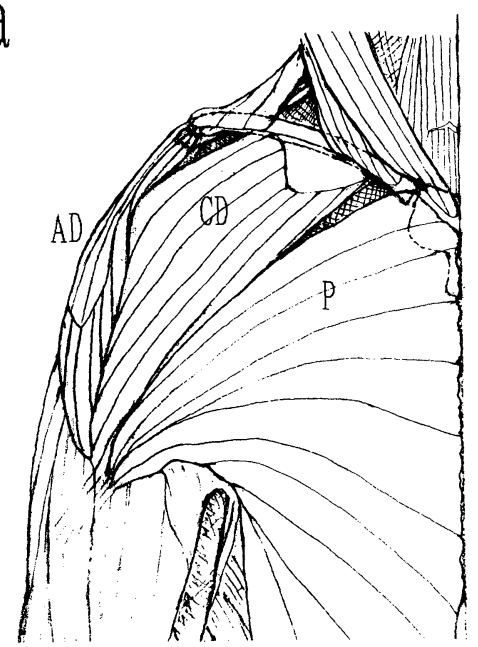

c

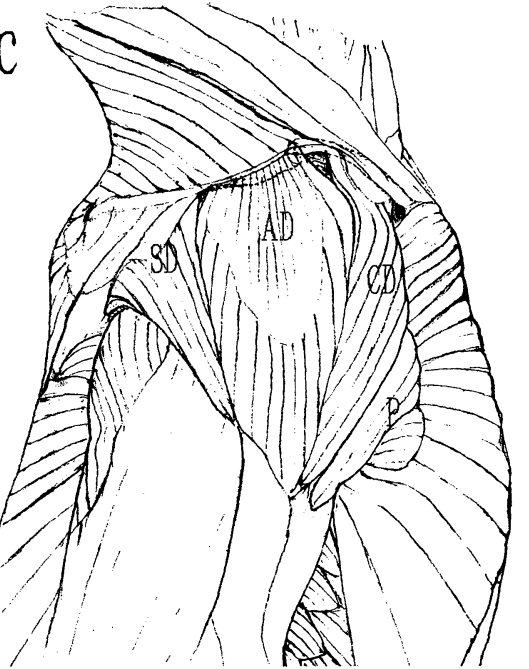

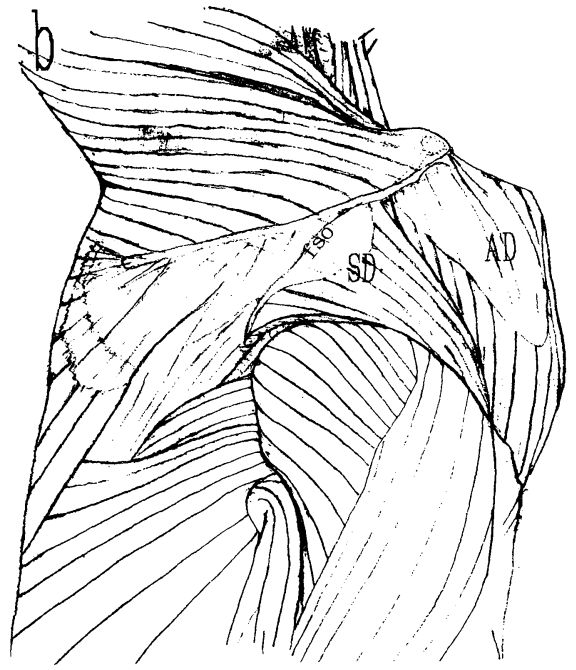

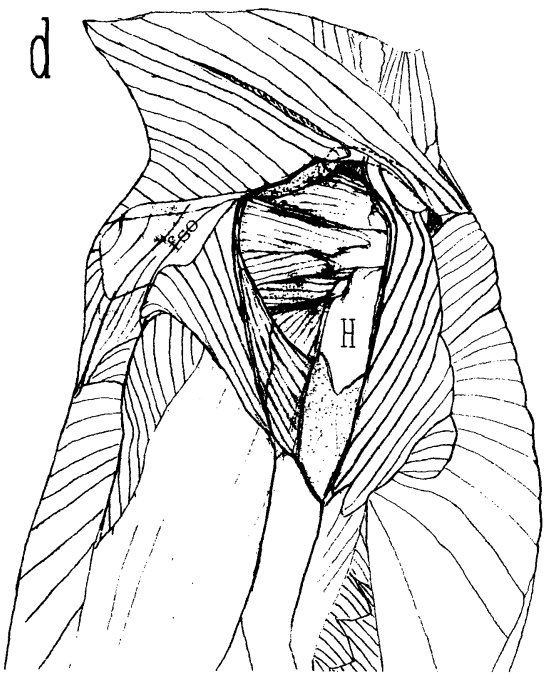

Fig. 1. Line drawings showing the topographic relations among the three component portions of the deltoid and their adjoining muscles in the Japanese macaque (Macaca fuscata). In Fig. 1d, the acromiodeltoid has been removed to show the insertions of the other two component portions. Note the consecutive arrangement observed within and among the component parts of the deltoid and pectoralis. For detail, see OBSERVATIONS. $\boldsymbol{A D}, \boldsymbol{C D}$, and $\boldsymbol{S D}$, acromio-, cleido-, and spinodeltoid, respectively; $\boldsymbol{P}$, pectoralis; $\boldsymbol{H}$, humerus; $\boldsymbol{f s o}$, functionally significant origin of the spinodeltoid. a, Ventral, $\mathbf{b}$, Laterodorsal, $\mathbf{c}$ and $\mathbf{d}$, Lateral view; all drawn on the same scale. The humerus is shown in its resting position.

fibres insert by fleshy fibres widely upon the distal half of the deltoid crest covering the region between 24 to $45 \%$, while the remainder (dorsal third) insert by way of the common tendon plate, as stated above. Near the insertion, the fibres in the 
a

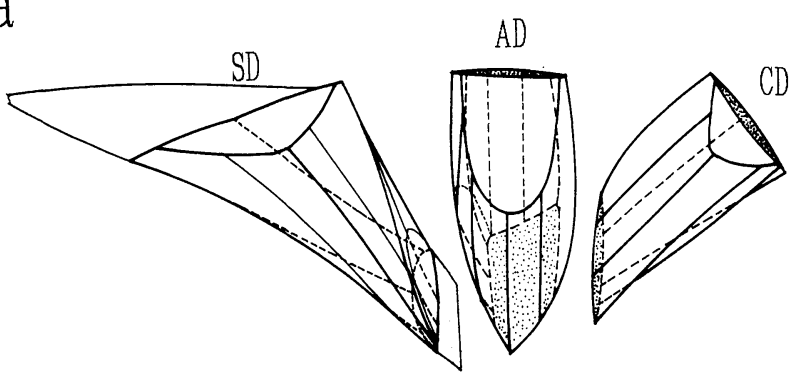

$\mathrm{b}$

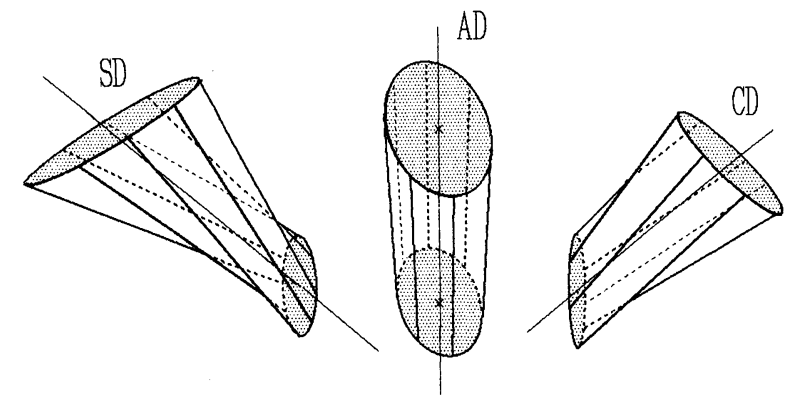

Fig. 2. Schematic drawings showing the spatial fibre architecture of the three component parts of the right deltoid muscle. The more superficially originating fibres in each part tend to reach more distal site upon the humeral insertion, although the degree of fibre radiation among the three differs. In $\mathbf{b}$, the fibre architecture is drawn more simply than in $\mathbf{a}$ in order to facilitate comprehension.

deep aspect of the muscle belly tend to converge again slightly, creating several short tendinous fibres. This muscle in the macaque, however, can be basically regarded as parallel-fibred, unlike the multi-pennate acromiodeltoid noted in human anatomical texts (see e.g., Williams et al., 1989, p. 613).

\subsection{Fibre architecture of the three deltoid muscles}

The following architectural features were recognized in the three portions of the deltoid:

1. The inner architecture of the acromiodeltoid indicates a unipennate muscle with nearly parallel fibres. The more superficial fibres attach to more distal sites on the humerus, connecting between the broad tendon plate at origin and the deltoid crest (Fig. 2a, $\boldsymbol{A D}$ ). Thus, this muscle can be classified as having a parallel-fibred, obliquely-cut cylinder shape (Fig. 2b, $\boldsymbol{A D}$ ).

On the other hand, the fibres of the other two deltoids (especially the spinodeltoid) tend to radiate. The tendon plates, which are relatively small, are negligible (Fig. 


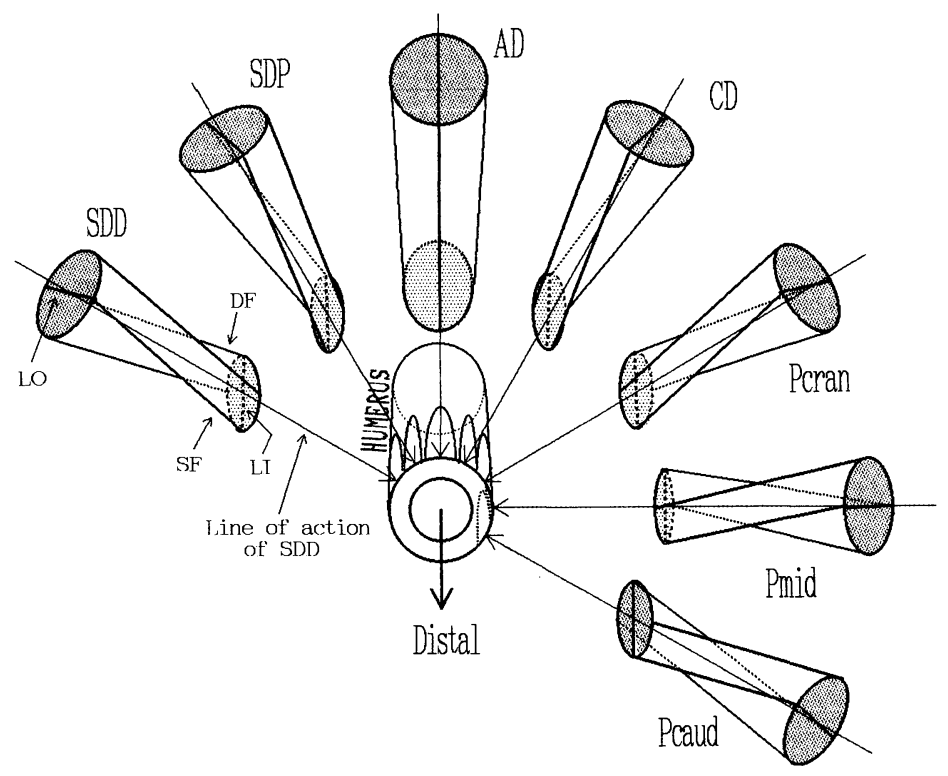

Fig. 3. Spatial orientation of the component bundles of $\mathrm{Mm}$. deltoideus and pectoralis. Component bundles of the two muscles are successively arranged both in origin and in insertion. From estimating the angle formed between the two lines (e.g. $\boldsymbol{L O}$ and $\boldsymbol{L I}$ ), each linking two arbitrarily selected fibres (e.g. $\boldsymbol{S F}$ and $\boldsymbol{D F}$ ) in the bundle, the following conclusions are reached: first, the degree of fibre twist gradually increases as the bundles originate further from the acromiodeltoid, reaching a maximum in the middle portion of the pectoralis and then decreasing; second, the direction of twist in the spinodeltoid is opposite that in the cleidodeltoid and the pectoralis. Right side, lateroventral view. Humerus is shown in its resting position. $\boldsymbol{A D}$, acromiodeltoid; $\boldsymbol{C D}$, cleidodeltoid; $\boldsymbol{D F}$ and $\boldsymbol{S F}$, the deepest and the most superficial fibre arising in the origin, respectively; $\boldsymbol{L I}$ and $\boldsymbol{L O}$, line linking the two fibres $(=\boldsymbol{S F}$ and $\boldsymbol{D F})$ in the insertion and in the origin, respectively; Pcran, Pmid, and Pcaud, cranial, middle, and caudal bundle of pectoralis, respectively; $\boldsymbol{S D D}$ and $\boldsymbol{S D P}$, distal and proximal bundle of spinodeltoid, respectively.

2a, $\boldsymbol{S D}$ and $\boldsymbol{C D}$ ). Thus, these two muscles can be classified as having a radiatingfibred cone shape (Fig. 2b, $\boldsymbol{S D}$ and $\boldsymbol{C D}$ ). However, the fibre architectural features of the two muscles can be basically thought of as identical to that of the acromiodeltoid, with the exception of the radiation.

2. All three portions are composed of numerous fibre bundles that arise successively along the line of origin, and attach around the humerus (e.g., Fig. 3).

By arbitrarily chosing two fibres which are running symmetrically about the main axis of the bundle (e.g., $\boldsymbol{S} \boldsymbol{F}$ and $\boldsymbol{D F}$ in Figs. 3 and 4), the degree of fibre twisting within the bundle can be defined as an angle formed by projecting the two lines that linking each fibres at origin and at insertion ( $\boldsymbol{L O}$ and $\boldsymbol{L I}$ in Figs. 3 and 4).

Using this method, the following conclusions can be drawn (Fig. 3): With the anatomical position of the forelimb used in the present study $(=$ at the resting 


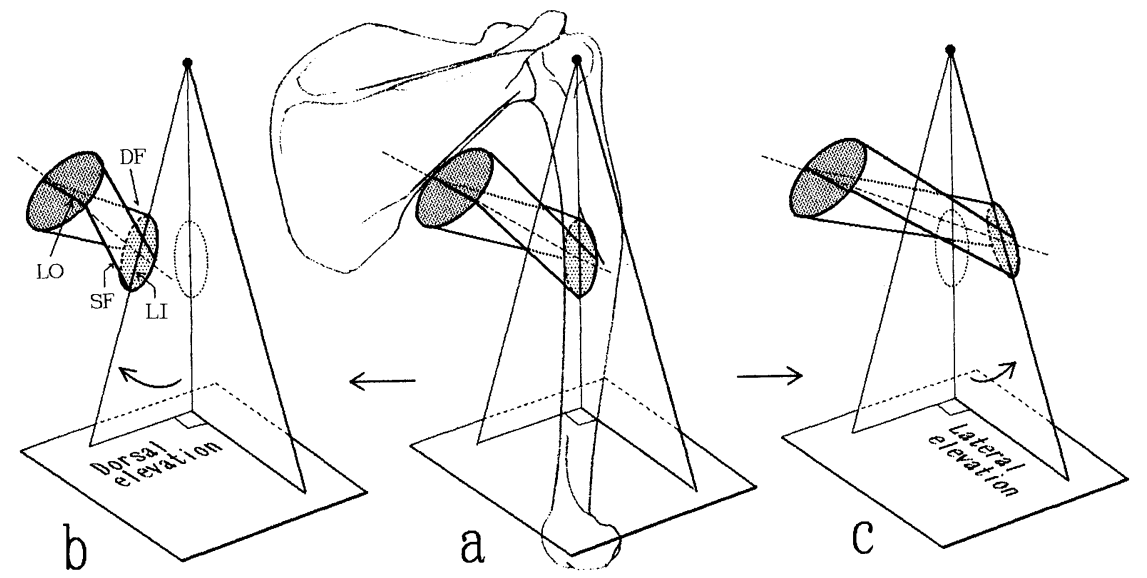

Fig. 4. Fibre architectural change in the distal bundle of spinodeltoid with the positional change of the humerus. Note the change in the angle formed between $\boldsymbol{L O}$ and $\boldsymbol{L I}$; The degree of fibre twist in the bundle gradually reduces when the humerus is laterally elevated (e.g., by the acromiodeltoid) from the resting position (c), while it remains largely unchanged when the bundle itself contracts and elevates the humerus dorsally from the same position (b). For detail, see DISCUSSION.

position of the humerus), fibre twisting in the acromiodeltoid is scarcely discernable. Within the other two deltoids, the degree of twisting increases in bundles emerging further from the acromiodeltoid. In addition, the fibres in the cleidodeltoid and the spinodeltoid twist in opposite directions. Thus, it becomes apparent that the fibres of these two muscles are arranged symmetrically about the middle fibres of the acromiodeltoid. Furthermore, it becomes clear that the degree (or direction) of fibre twisting in a given bundle in the deltoid (even in the acromiodeltoid) will vary (or reverse) in accordance with the change of the spatial position of the humerus relative to the shoulder girdle; for instance, when the humerus which is at first flexed forwardly (= ventrally) becomes then extended backward (= dorsally), the direction of twisting in the acromiodeltoid will reverse.

\section{DISCUSSION}

\subsection{Functional implications of fibre twisting within a bundle}

When the humerus is placed at its resting position, well-marked twisting within the bundles can be observed, particularly within bundles of the spinodeltoid running near the axillary border. The author will discuss here the functional implications of this structure, adopting $S D D$ in Figs. 3 and 4 as an example.

First, owing to the presence of twisting, each fibre composing SDD have variable insertions (via a tendon plate) on the humerus: the more superficial fibres 
attach to more distal sites on the humerus at smaller angles (Fig. 4a). Nevertheless, the mechanical action exerted by the individual fibres is essentially the same: any fibre in the SDD acts to elevate the humerus in the manner of a 3rd-class lever whose fulcrum is at the humeral head. The resultant force exerted by the fibres in a given contractile fibre unit with a geometrically simple shape can be considered to act along a line defined by the loci of the centroids of its transverse cross sections, if the fibres of the unit are distributed evenly over the cross sectional area (Jensen and Davy, 1975; Koolstra et al., 1989). Thus, the line of action in SDD coincides with its main axis, or the central fibre in the bundle (See also the next section). Accordingly, it is apparent that the humerus can never be rotated around this line but can only be elevated along this line by the simultaneous contraction of its component fibres.

Second, the method described above allows us to theoretically estimate that the degree of twist in SDD gradually decreases when the acromiodeltoid elevates the humerus laterally from its resting position (Fig. 4c). In contrast, the degree of twist barely changes when the SDD contracts itself and elevates the humerus dorsally from the same position (Fig. 4b).

Hence, it becomes evident that the twisting structure itself exerts no torsional influence on the elevation of the humerus, nor does the fibre contraction in a twisting bundle produce any special effect that reduces its own degree of twist. Accordingly, the law of detorsion, which states that those muscles that are twisted at rest will induce torsional forces when stimulated (MacConaill and Basmajian, 1969; Gans, 1982), is not applicable to the deltoid muscle when we take into account the mobility characteristic of the shoulder joint.

\subsection{Evolutionary formation of the twisting structure}

Twisting within bundle is observed not only in the deltoids but in the pectoral muscles as well. In the latter, however, the crossing of bundles among composing muscle parts is pronounced (cf. Fujino et al., 1990). When the forelimb is held at the resting position, the degree of twist within a bundle increases in both muscles as the bundle arises more distantly from the acromion (Fig. 3). In function, however, this structure within the pectoral muscles may produce direct rotation of the humerus around the centre of the inserting region, unlike that in the deltoid.

On the other hand, Horejsi (1967) has briefly reported in his human embryological study that the fibre bundles of the spinodeltoid take on a parallel arrangement at first attaching via a tendon plate upon the humerus. However, the entire structure, including the tendon, then supinates during development and a twisted structure is formed. Further, he pointed out that the developmental process of the twisted structure in the spinodeltoid is analogous to that observed by Cihak (1959) in the component parts of the pectoralis major. Although he only observed the structure 
in the spinodeltoid, the developmental process there strongly suggests that a similar modification (i.e., rotation of bundles on the insertion side) should also occur in the cleidodeltoid, which possesses the same structure. Again, this modification observed by Horejsi in the spinodeltoid is in fact analogous to the mechanism that causes the bundles in the mammalian pectoral muscles to cross as well as the fibres within each component part of them to twist (cf. Fujino et al., 1990).

These findings allow the direct cause for the twisted bundle structure of both muscles to be explained as follows (Fig. 5):

In evolutionary development toward the therian mammals, the humerus has gradually become oriented more perpendicularly to the horizontal plane (Gregory, 1949). Thus, it is most likely that the spatial orientation of the humerus relative to the thorax or the shoulder girdle that allows a bundle or muscle part connecting the humerus with the thorax to exert the most significant action has also changed, even though the same muscle origin and insertion has been retained.

In a symmetrically-shaped muscle whose fibres are arranged in a symmetrical pattern and distributed evenly over its cross-section (e.g., a muscle with a parallelfibred cylinder shape such as the acromiodeltoid, or a muscle with a radiatingfibred cone shape such as the spinodeltoid; see Fig. 2b), the line of action will coincide with the main axis (Jensen and Davy, 1975; Koolstra et al., 1989) (cf. the

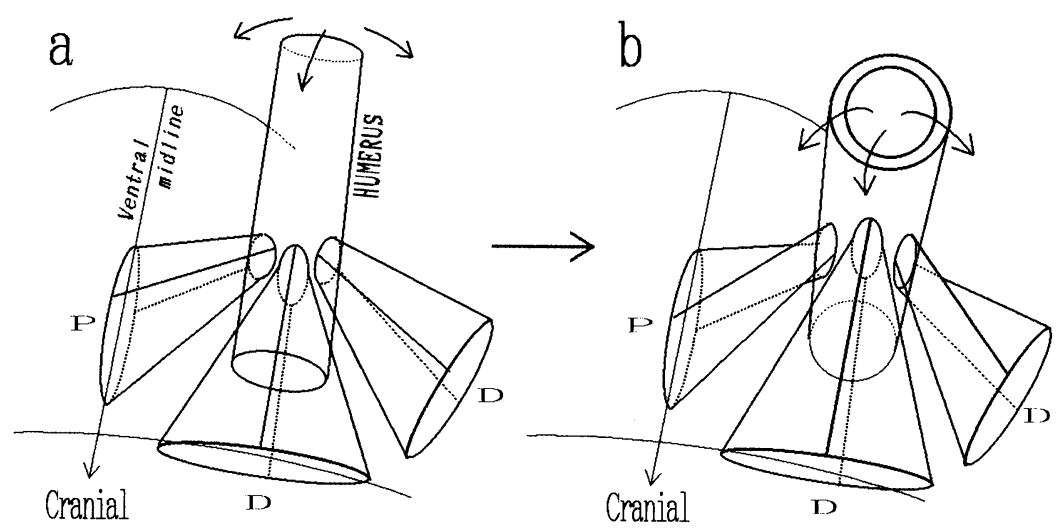

Fig. 5. Evolutionary relations between the muscle fibre architecture and the spatial orientation of the humerus. In the reptilian condition (a), the humerus is held almost parallel with the horizontal plane. In all bundles except those arising from the cranial part of the shoulder girdle, the fibres arising further from the part insert upon the more distal sites on the humerus. In contrast, in the mammalian condition (b) where the humerus is held perpendicularly to the thorax, fibres in all bundles are arranged so that the more superficial fibres insert upon more distal sites; thus, when the humerus is held, for instance, at the resting position, fibre twisting is inevitable in every bundle except those of the acromiodeltoid. Right side, cranoventral view. $P$ : pectoral muscle; $\boldsymbol{D}$ : deltoid muscle. For detail, see DISCUSSION. 
preceding section). To put it differently, the fibres composing a muscle must be ideally arranged, in an adaptive sense, in a symmetrical pattern about a line linking the origin and the insertion of the muscle when the spatial orientation of the insertion relative to the origin (e.g., the posture of the humerus relative to the thorax) is most significant for the animal.

In reptiles, the humerus is aligned almost parallel to the long axis of the thorax. The desired action for a contractile unit arising some distance from the cranial portion of the shoulder girdle is to adduct or to abduct the humerus in an almost horizontal plane (Fig. 5a). Accordingly, the optimal arrangement for the fibres composing the unit, in a mechanical sense, is a symmetrical pattern about the line of action linking the horizontally-placed humerus and the thorax or shoulder girdle. As a result, the more distantly-arising fibers in the unit will insert upon more distal sites on the humerus. In contrast, in the therian mammals, in which the humerus is virtually perpendicular to the thorax, the optimal fibre arrangement is a symmetrical pattern about the line of action linking the perpendicularly-disposed humerus and the thorax or shoulder girdle. Thus, the most superficial fibres in a contractile unit insert upon the most distal sites on the humerus (Fig. 5b).

Hence, in mammals, the twisted bundle structure is inevitable unless the long axis of the humerus is placed perpendicular to the line along which the bundle originates (as is the case with the acromiodeltoid when the humerus is held in the scapular plane, cf. Fig. 3, $A D$ ).

The twisting structure can therefore be considered to have been formed concomitantly with a fibre architectural modification in which the component bundles preserved their maximum efficiency in operating the humerus, through the evolutionary postural change of the humerus from the reptiles to the therian mammals.

When this modification occurs simultaneously in the radiating bundles which successively arise along the line of origin, their insertions inevitably become arranged in a multi-stratified manner consecutively surrounding the anterior half of the humerus (Figs. 3, 5b). This can be considered to be the precise cause for the overlapping structure often observed in the mammalian deltoids and pectorals, both individually and between them (cf., e.g., Parsons, 1898). Again, in a distantlyarising muscle as well as in its component bundles, one can clearly see that the fibres arising at a greater distance from the acromion have gradually reached deeper and more proximal site in its insertion, and vice versa, as a result of this modification. (The intermediate condition between Figs. $5 \mathrm{a}$ and $5 \mathrm{~b}$ is not shown, but may easily be imagined by the reader.) This strongly indicates that the peculiar architecture of the current pectoral muscles in mammals (i.e., the presence of mutually-crossing bundles) has been formed at least in part through this modification (cf. Fujino et al., 1990; see also the next section). 


\subsection{Structural differentiation between the deltoids and the pectorals}

In attempting to discern the factors affecting structural differences in the fibre architecture of the two muscles, the author first will discuss their morphological relations in insertion here, and those in origin in the next section, taking into account their evolutionary background.

In primitive reptiles such as Sphenodon (cf. Fürbringer, 1900), the elements of the pectoral girdle are so firmly attached to one another that there can be very little movement of the girdle relative to the thorax. Hence, the radiating and mutually contiguous muscles of both the deltoid and pectoralis (the former is termed $M$. acromialis by Ribbing, 1938) each act simply to operate the humerus relative to the thorax, similar to a 3rd-class lever whose fulcrum is at the humeral head. The direction of movement is the only difference, i.e., the former acts to protract or elevate the humerus while the latter acts to adduct or depress it (Fig. 5a).

As mammal-like reptiles evolved toward the therian mammals, the bony elements composing the pectoral girdle became markedly reduced, or disappeared. As a result, current mammals have come to possess the shoulder girdle and the humerus that are significantly more mobile relative to the thorax and shoulder girdle, respectively (Kemp, 1982). In response to this remodelling, the pectoral muscle also can be considered to have been fundamentally modified, through the formation of the crossing structure, into several component parts which distinctly differ in function: the cranial portion for elevation of the humerus, the middle for adduction, and the caudal for retraction (Fujino et al., 1990). Despite the fact that the deltoids underwent the architectural modification stated in the preceding section, it basically retains its primitive structure and function: no crossing bundles are present, and all bundles basically insert on the similar level of the humerus (cf. OBSERVATIONS).

The presence or lack of mutually-crossing bundles in these two muscles is most likely attributable to the fundamental difference in function between the articulations over which each muscle passes. These are the shoulder joint in the broad sense (the glenohumeral for the lateral two deltoids, and the glenohumeral plus acromioclavicular joints for the cleidodeltoid), and the thoraco-humeral joint, which is composed of the above two plus the sternoclavicular joints for the pectoral muscle (cf. Fig. 6). This is because the former limits the deltoid to acting simply as a 3rd-class lever (although two joints are interposed between the origin and the insertion of the cleidodeltoid, it actually behaves just like one), whereas the latter allows the pectoral muscle to perform much more complex movements. Consequently, its morphology and function are both differentiated, probably through the fibre architectural modification discussed in the preceding section.

In fact, the nature of the shoulder joint is such that shifting the insertion of the deltoid proximally, like that of the caudal bundles of the pectorals, would seem to 
have no mechanical significance*. Hence, a potential and mechanically significant modification for the deltoid would be to extend or shift its origin, and thus to enlarge the range or change the direction in which it can operate the humerus. Further, O'Brien et al. (1990), following Inman et al. (1944), have pointed out that in response to the functional demands of orthograde posture certain peculiar changes have occurred in the primate evolution regarding the deltoid: 1 . distal migration of the site of insertion, 2. increase in its mass, and 3. enlargement of the acromion. These changes have increased its mechanical advantage in shoulder motion. Actually, the first two modifications also serve to increase the mechanical advantage of the cranial portion of the pectoralis adjoining the deltoid by a nearly equivalent amount. This is probably another reason why this portion of the pectoralis has experienced modifications similar to those in the deltoid. In contrast, the caudal pectoral muscles have been differentiated virtually into muscles that work to retract the shoulder joint, by shifting insertion cranially (Fujino et al., 1990). This probably has concomitantly allowed the humerus a wider range of motion; this functional significance was suggested by Mörike (1954) regarding the pectoralis major muscle.

In addition, it is widely known that the human acromiodeltoid is multi-pennated (cf., e.g., Williams et al., 1989). Embryological study by Horejsi (1967) revealed that the human acromiodeltoid is initially parallel-fibred, but gradually becomes multi-pennated as it develops. Hence, at least in the primates, the formation of multi-pennated structure may be considered a modification to elevate the forelimb more powerfully toward the human erect posture. The parallel-fibred acromiodeltoid observed in the macaques may reflect its restricted role in their pronograde posture.

\subsection{Morphological relations between the cleidodeltoid and the clavicular portion of pectoral muscle}

The close topographical relations between the two muscles noted earlier (cf. INTRODUCTION) appear to have been essentially maintained in the therian mammals (cf., e.g., Leche, 1874-1900). It appears, however, certain peculiar modifications have further occurred in the muscle origins:

1. While the origin of the pectoralis still tends to be basically limited cranially up to about the sternoclavicular joint, the medial territory of the origin of the cleidodeltoid tends to disappear on the clavicle. Thus, an open space between the origins of the two muscles is often formed (this is observed in the marsupial opossum, cf. Coues (1872), and also in most prosimians, cf. Jouffroy (1962), p. 51) (cf. Fig. 6a-2).

* An embryological study of the opossum shows that M. teres minor, an early separator from the deep portion of the deltoid proper, establishes its insertion upon the greater tuberosity before the deltoid proper reaches its insertion (Cheng, 1955); thus it will act exclusively as a fixator of the shoulder joint. 

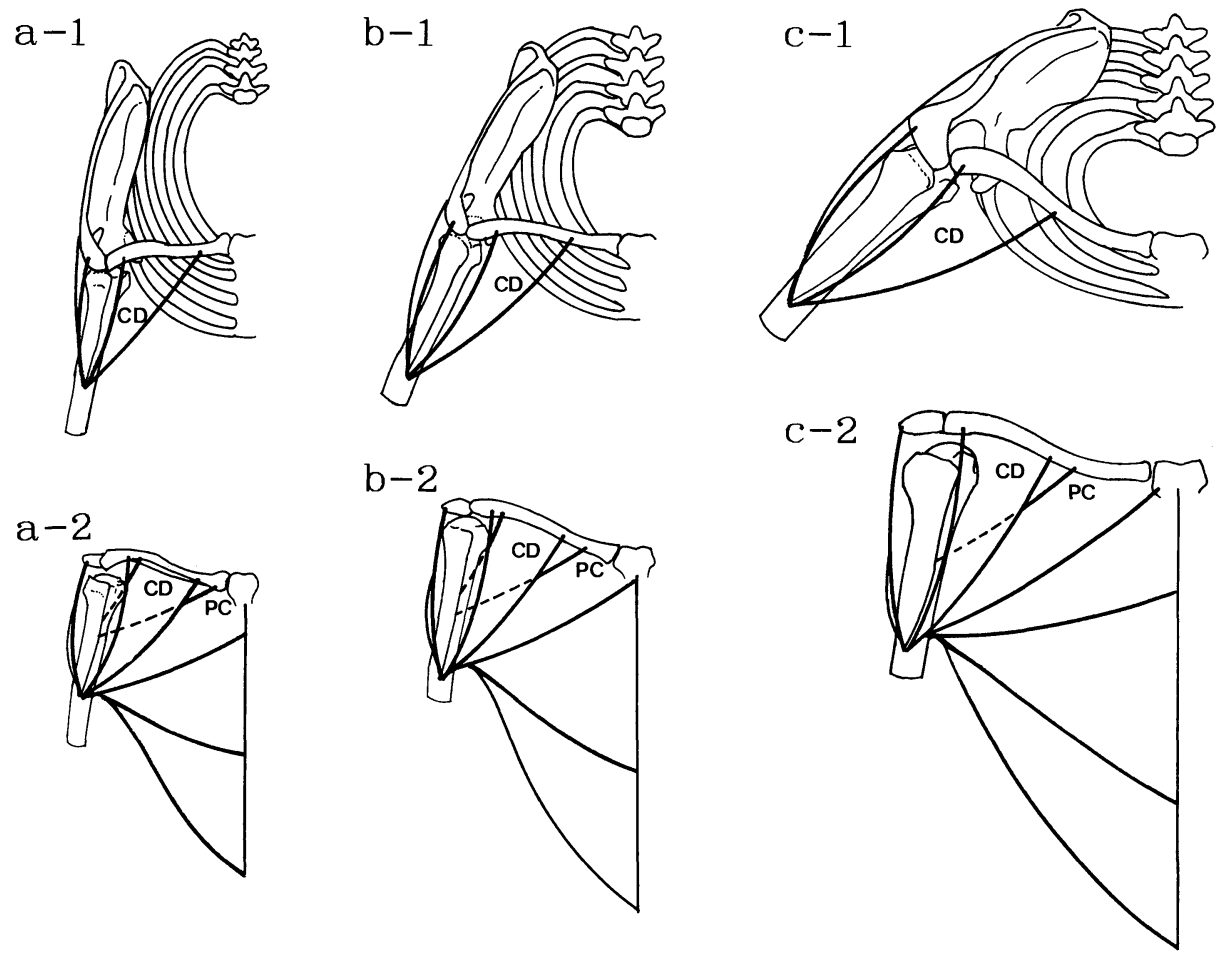

Fig. 6. Morphological relations between the cleidodeltoid and the clavicular portion of the pectoral muscle in primate evolution. As an evolutionary trait characteristic of brachiating locomotion or human orthograde bipedalism, the thorax has gradually become relatively flatter in the anteroposterior dimension, and concomitantly the clavicle has become elongated relative to the scapular dimension. As a result, the relative extent of the cleidodeltoid to the clavicle has been reduced and thus the muscle appears to diminish toward the acromion (a-1, b-1, c-1). The pectoralis in some brachiators and in humans has extended onto the clavicle, possibly to maintain or to increase the mobility of the humerus in the forward and horizontal flexions, probably with the cleidodeltoid acting synergistically (a-2, b-2, c-2). $\mathbf{a}, \mathbf{b}$, and $\mathbf{c}$, macaque, intermediate, and human condition, respectively; $\boldsymbol{C D}$, cleidodeltoid; $\boldsymbol{P C}$, the most cranial bundle of the pectoralis. (a-1, b-1, and c-1 modified after O'Brien et al., 1990)

2. Further, at least in placental claviculate mammals, significant clavicular origin of the pectoralis has been often reported, e.g., in Chiroptera, it arises from the sternal half to three-quarters of the clavicle (Saar, 1903; Vaughan, 1959; Strickler, 1978); in the rat, from the sternal third (Hebel and Stromberg, 1986); in some (semi-)brachiating primates, such as howling and woolly monkeys, the great apes except for the orang, and in bipedal humans, and especially in the gibbon and siamang, it arises from the sternal two-thirds (Campbell, 1937; Kallner, 1956; Ashton and Oxnard, 1963; Preuschoft, 1965; Schön, 1968; Stern et al., 1980). Along with such lateral extension of the pectoralis onto the clavicle, the cleidodeltoid in 
these animals generally tends to arise less broadly from its lateral part than in animals with no clavicular origins of the pectoralis. Thus, the border zone on the clavicle between the two muscles has come to shift laterally in these animals (cf. Figs. 6b-2, 6c-2).

In function, whether the cleidodeltoid or the clavicular pectoralis extends more broadly upon the clavicle, the total action exerted by the two upon the humerus can be expected to be the same, if they insert on the same level. In fact, their synergistic action (synchronously active electromyography) has been confirmed for the forward flexion of the human forelimb (Inman et al., 1944).

These facts raise the question: why has the pectoralis extended onto the clavicle in these animals?

Stern et al. (1980) have put forward an explanation for this modification in primates, noticing that among non-human primates only those with cranially-displaced shoulder joints possess a significant clavicular origin. They inferred that such formation may enhance or compensate for the forelimb flexion exerted by the sternocostal pectoralis, in the above remodelling (= cranial displacement) of the shoulder. As they admit, however, this concept cannot directly account for the absence of clavicular origin in the spider monkey and orang, both of which have cranially-displaced shoulder joints, nor for the presence of extensive clavicular origin (arising from the sternal half) in humans, who have shoulder joints on the same level as the manubrium.

On the basis of the topographical and functional relations between the two muscles, the author will now offer another possible explanation for the extension of the clavicular origin of pectoralis, at least in primates:

1. Macaques retain an excursion pattern of scapula similar to that of other quadrupedal mammals, with the scapula placed on the lateral aspect of the relatively narrow thorax (Jenkins, 1974; Jenkins et al., 1978) (Fig. 6a-1). Hence their shoulder girdle and thorax can be considered to be primitive (= non-specialized) relative to other primates. Likewise, the muscular structure in macaques can also be considered primitive: the pectoralis is limited cranially upon the manubrium, whereas the cleidodeltoid arises broadly from the clavicle (from its middle half to threefifths, cf. OBSERVATIONS). Thus, in macaques the humeral elevation in the scapular plane exerted by deltoids virtually coincides with that in the parasagittal plane.

2. The thorax has gradually experienced a relative flattening in the anteroposterior dimension, as one of the evolutionary traits toward brachiating locomotion, or the human orthograde posture. Concomitantly, the scapula has become placed on the more dorsal aspect of the thorax, and the clavicle, which spans the distance between the manubrium and the acromion, has also become elongated relative to the scapular dimension (Erikson, 1963; Ashton and Oxnard, 1964; Jenkins et al., 1978) 
(Figs. 6b-1, 6c-1).

Through this remodelling, the deltoid muscle has also gradually shifted laterodorsally, wrapping the shoulder joint. Thus, the line of total action supplied by the deltoid's three parts has become directed more laterally, since the deltoid as a whole is thought to be optimally aligned with the elevation of the humerus in the scapular plane (this has been confirmed in humans, cf. Zuckerman and Matsen, 1989).

3. When it is necessary for the deltoids to retain the equivalent ability to elevate the humerus in the scapular plane through this bony remodelling, it becomes theoretically necessary for the cleidodeltoid to maintain the equivalent width of its origin on the clavicle. Consequently, the relative extent of the cleidodeltoid to the clavicle has reduced and thus the muscle appears to diminish toward the acromion. This reduction of the bundle connecting the medial region of the clavicle with the humerus naturally leaves less force available to elevate the humerus cranomedially. It is therefore possible that the pectoralis has extended so far onto the clavicle in response to a functional demand to maintain or to increase, probably with the cleidodeltoid acting synergisticly, the mobility of the humerus in forward (and most likely horizontal) flexion. In brief, it is the deltoid that is primarily modified with the flattening of the thorax, and the pectoralis secondarily extends onto the clavicle to supplement, when necessary, the relatively diminished cleidodeltoid upon the elongated clavicle. In order to verify this hypothesis, the ability of the two muscles to operate the humerus should be quantitatively analysed, using animals from several different taxa, in light of other aspects of fibre architecture such as fibre length, cross sectional area, etc. Further study in this area will be performed.

\section{SUMMARY}

1. The deltoid fibre bundles are twisted like those of the pectorals, but do not have the mutually-crossing structure of the pectorals.

2. The deltoid fibre bundles are arranged symmetrically about the middle fibre of the acromiodeltoid, i.e., the degree of fibre twist increases in bundles that arise further from the acromion, while the bundles in the spino- and cleidodeltoid twist in opposite directions.

3. The twisted fibre structure of the deltoid bundles is unable to exert any torsional action upon the humerus, unlike that of the pectorals.

4. The twisted fibre structure within both the deltoid and pectoral bundles has been formed through an evolutionary architectural modification in the mammallike reptiles and primitive mammals, which allows the humerus to operate in the mechanically most efficient manner as it became oriented more perpendicularly to the trunk. 
5. The structural differentiation between the two muscles has been probably caused by the functional difference in the respective articulations interposed between their origins and insertions.

6. As a result of the relative reduction of the cleidodeltoid upon the clavicle concomitant with the evolutionary flattening of the thorax in the primates, the cranomedial elevation of humerus has probably become weakened; the pectoralis in some brachiators and in humans has extended onto the clavicle possibly to supplement this action.

\section{ACKNOWLEDGEMENT}

Five Japanese macaques were obtained from the Primate Research Institute, Kyoto University, through Dr. T. Kimura. The present work was supported, in part, by a grant through the collaboration research system of PRI, Kyoto University, and also by a grant-in-aid for scientific research (No. 04304010) from the Japanese Ministry of Education, Science, and Culture.

\section{REFERENCES}

Ashton, E.H., and Oxnard, C.E. (1963) The musculature of the primate shoulder. Trans. Zool. Soc. Lond. 29, 553-650.

Ashton, E.H., and Oxnard, C.E. (1964) Functional adaptations in the primate shoulder girdle. Proc. Zool. Soc. Lond. 142, 49-66.

Campbell, B. (1937) The shoulder musculature of the platyrhine monkeys. J. Mammal. 18, 66-71.

Cheng, C.C. (1955) The development of the shoulder region of the opossum, Didelphys virginiana, with special reference to the musculature. J. Morphol. 97, 415-471.

Cihak, R. (1959) Musculus pectoralis major und seine Komponenten in der Ontogenese des Menschen. Cescoslovenska Morphologie 7, 174-191. (In Czeck with Russian and German abstracts)

Coues, E. (1871) On the myology of the Ornithorhynchus. Communications Essex Institute 6, 127-173.

Coues, E. (1872) The osteology and myology of Didelphys virginiana. Memoirs Bost. Soc. Nat. Hist. 2, 41-154.

Erikson, G.E. (1963) Brachiation in new world monkeys and in anthropoid apes. Symposia Zool. Soc. Lond. 10, 135-163.

Fujino, K., Kuramoto, K., and Mochizuki, K. (1990) Spatial fibre architecture of Mm. pectorales in macaques: their morphological, phylogenetical, and functional implications. Gegenbaurs Morphol. Jahrb. 136, 587-602.

Fürbringer, M. (1990) Zur vergleichenden Anatomie des Brustschulterapparates und der Schultermuskeln. IV. Teil., Jena, Z. Nat. Forsch. 34, 215-718 (pp. 471-474 for Pectoralis, pp. 482486 for Deltoidei of Sphenodon; see also Figs. 161-170 in Taf. 16.).

Gans, C. (1982) Fiber architecture and muscle function. Exerc. Sport. Sci. Rev. 10, 160-207.

Gregory, W.K. (1949) The humerus from fish to man. Amer. Mus. Novitates 1400, 1-54.

Hebel, R., and Stromberg, M.W. (1986) Anatomy and Embryology of the Laboratory Rat, BioMed Verlag, Worthsee.

Horejsi, J. (1967) Ontogenesis of external deltoid muscle formation in man. Acta Unversitatis Carolinae 
Medica 13 (1), 81-91.

Howell, A.B. (1936) The phylogenetic arrangement of the muscular system. Anat. Rec. 66, 295-316.

Howell, A.B., and Straus, W.L., Jr. (1961) The muscular system. In The Anatomy of the Rhesus Monkey (Hartman, C.G., and Straus, W.L., Jr., ed.), Hafner, New York, pp. 89-175 (Reprint edition, originally published in 1933 by Williams \& Wilkins).

Inman, V.T., Saunders, J.B. deC. M., and Abbott, L.C. (1944) Observations on the function of the shoulder joint. J. Bone Joint Surg. 26A, 1-30.

Jenkins, F.A., Jr. (1974) The movement of the shoulder in claviculate and aclaviculate mammals. $J$. Morphol. 144, 71-83.

Jenkins, F.A., Jr., Dombrowski, P.J., and Gordon, E.P. (1978) Analysis of the shoulder in brachiating spider monkeys. Amer. J. Phys. Anthropol. 48, 65-76.

Jensen, R.H., and Davy, D.T. (1975) An investigation of muscle lines of action about the hip: A centroid line approach vs. the straight line approach. J. Biomechanics 8, 103-110.

Jouffroy, F.K. (1962) La musculature des membres chez les lémuriens de Madagascar. Etude descriptive et comparative. Mammalia 26 (Suppl. 2), 1-326.

Kallner, M. (1956) Die Muskulatur und die Funktion des Schultergürtels und der Vorderextremität des Orang-Utans. Morphol. Jahrb. 97, 554-665.

Kemp, T.S. (1982) Mammal-like Reptiles and the Origin of Mammals, Academic Press, London.

Koolstra, J.H., Eijden, T.M.G.J., van, and Weijs, W.A. (1989) An iterative procedure to estimate muscle lines of action in vivo. J. Biomechanics 22, 911-920.

Leche, L. (1874-1900) Musculatur. In Bronn's Klassen und Ordnungen des Thier-Reichs, 6. Band., V. Abt., Säugethiere: Mammalia., Band 1. (pp. 758-766 for Mm. pectorales; pp. 791-794 for M. deltoideus.)

MacConaill, M.A., and Basmajian, J.V. (1969) Muscles and Movements: A Basis for Human Kinesiology, Williams and Wilkins, Baltimore (cited from Gans, 1982).

McKay, W.J.S. (1895) The morphology of the muscles of the shoulder-girdle in monotremes. Proc. Linn. Soc. New South Wales 9, (1894), 263-360.

Mörike, K.D. (1954) Vergleichend-functionelle Morphologie der Rumpf-Oberarmmuskulatur der Säugetiere und des Menschen. Morphol. Jahrb. 94, 165-237.

O’Brien, S.J., Arnoczky, S.P., Warren, R.F., and Rozbruch, S.R. (1990) Developmental anatomy of the shoulder and anatomy of the glenohumeral joint. In The Shoulder (Rockwood, C.A., Jr., and Matsen, F.A., III, ed.), Vol. 1, W.B. Saunders, Philadelphia, pp. 1-33.

Parsons, F.G. (1898) The muscles of mammals, with special relation to human myology. Lecture II. - The muscles of the shoulder and forelimb. J. Anat. Physiol. 32, 721-752.

Preuschoft, H. (1965) Muskeln und Gelenke der Vorderextremität des Gorillas (Gorilla gorilla Savage et Wyman, 1847). Morphol. Jahrb. 107, 99-183.

Ribbing, L. (1938) Die Muskeln und Nerven der Extremitäten. In Handbuch der vergleichenden Anatomie der Wirbeltiere (Bolk, L., Göppert, E., Kallius, E., und Lubosch, W., ed.), 5. Band, Urban \& Schwarzenberg, Berlin und Wien, pp. 543-656.

Saar, G.F., von (1903) Zur vergleichenden Anatomie der Brustmuskeln und des Deltamuskels. Arch. Anat. Phys., Anat. Abt. 27, 153-204.

Sacks, R.D., and Roy, R.R. (1982) Architecture of the hind limb muscles of cats: Functional significance. J. Morphol. 173, 185-195.

Schön, M.A. (1968) The muscular system of the red howling monkey. United States Nat. Mus. Bull. 273, 1-185.

Stern, J.T., Jr., Wells, J.P., Jungers, W.L., Vangor, A.K., and Fleagle, J.G. (1980) An electromyographic 
study of the pectoralis major in Atelines and Hylobates, with special reference to the evolution of a pars clavicularis. Amer. J. Phys. Anthropol. 52, 13-25.

Strickler, T.L. (1978) Functional osteology and myology of the shoulder in the Chiroptera. In Contributions to Vertebrate Evolution (Hecht, M.K., and Szalay, F.S., ed.), Vol. 4, Karger, Basel, pp. 1198.

Vaughan, T.A. (1959) Functional morphology of three bats: Eumops, Myotis, Macrotus. University of Kansas Publications Museum of Natural History 12 (1), 1-153.

Westling, C. (1889) Anatomische Untersuchungen über Echidna. Bihang. Till K. Svenska Vet.-Akad. Handlingar. Afd. 4. No. 3, 15, 1-71.

Williams, P.L., Warwick, R., Dyson, M., and Bannister, L.H., ed. (1989) Gray's Anatomy, 37th ed., Churchill Livingstone, Edinburgh.

Zuckerman, J.D., and Matsen, F.A., III (1989) Biomechanics of the shoulder. In Basic Biomechanics of the Musculoskeletal System (Nordin, M., and Frankel, V.H., ed.), 2nd Ed., Lea \& Febiger, Philadelphia, pp. 225-247. 